\title{
Musique sertaneja, sonorités du quotidien et expériences corporelles au féminin (Goiás, Brésil)
}

Marina Rougeon

\section{(2) OpenEdition}

Édition électronique

URL : http://journals.openedition.org/pa/149

DOI : $10.4000 /$ pa. 149

ISSN : 2273-0362

\section{Éditeur}

Université Lumière Lyon 2

\section{Édition imprimée}

Date de publication : 31 octobre 2012

Pagination : 179-203

ISBN : 1634-7706

ISSN : 1634-7706

\section{Référence électronique}

Marina Rougeon, «Musique sertaneja, sonorités du quotidien et expériences corporelles au féminin (Goiás, Brésil) », Parcours anthropologiques [En ligne], 8| 2012, mis en ligne le 20 avril 2013, consulté le 02 mai 2019. URL : http://journals.openedition.org/pa/149 ; DOI : 10.4000/pa.149 


\section{Musique sertaneja, sonorités du quotidien et expériences corporelles au féminin (Goiás, Brésil)}

Marina Rougeon
Université Lumière Lyon 2, CREA

Partant d'éléments ethnographiques, cet article propose de réfléchir aux rapports entre les sonorités quotidiennes propres à certains quartiers brésiliens d'aujourd'hui et les modalités corporelles de déplacement des femmes dans ces espaces. L'ethnographie en question s'inscrit dans le cadre $\mathrm{d}^{\prime}$ une recherche doctorale en cours ${ }^{1}$. Elle se situe dans une région peu investie par l'anthropologie, le Centre Ouest brésilien, et plus spécifiquement dans certains quartiers de la ville de Goiás, les quartiers de João Francisco et Aeroporto, dans lesquels vit la plus grande partie des habitants ${ }^{2}$.

$C^{\prime}$ est la nécessité de penser ensemble les sonorités, les espaces et les expériences corporelles quotidiens qui sous-tend cette réflexion. L'idée d'ambiance développée par Jean-François Augoyard au sens de "fond du sensible " qui " naît de la rencontre entre les propriétés physiques environnantes, [la] corporéité avec sa capacité de sentir-se mouvoir et une tonalité affective » $(2007 / 2008: 60)$, me semble particulièrement opérante dans ce sens, dans sa dimension sonore. Elle sera donc au cœur de la problématique ici proposée. Comment les corps de femmes répondent à cette ambiance sonore et s'en imprègnent, participant ainsi de la construction d'un univers féminin par leur manière de se déplacer dans l'espace ?

Pour y répondre, je présenterai les sonorités du quotidien qui meuvent les quartiers João Francisco et Aeroporto, et en particulier la dimension musicale de ces ambiances sonores à l'articulation des espaces extérieurs et intérieurs, en privilégiant un genre musical particulier, les musiques sertanejas. Il sera

${ }^{1}$ Cette ethnographie a commencé en 2006 et a d'abord donné origine à un mémoire de Master en Anthropologie (Rougeon, 2008).

${ }^{2}$ Entre 3.000 et 5.000 sur 30.000 que compte la municipalité. Les nouveaux quartiers, dits périphériques, ont surgi dans les années 1960 quand Goiás a connu une expansion urbaine rapide avec la venue de migrants issus des États voisins et des zones rurales proches. Ils naissent dans un vaste contexte de modernisation et d'urbanisation de l'ouest brésilien. La révolution verte, la marche vers l'ouest, le «plan des objectifs » du gouvernement de Juscelino Kubitschek en 1956, qui avait pour slogan ambitieux " 50 ans de progrès en 5 ans », sont autant de projets politiques de développement économique mis en œuvre à l'époque par les dirigeants pour désenclaver les espaces alors jugés inoccupés de la plus grande partie du pays, du Centre-Ouest à l'Amazonie. 
ensuite question de souligner le rapport entre sonorités, écoute et réponses par le corps à ces musiques de la part des femmes pour comprendre l'existence de véritables chorégraphies de la séduction alimentant un univers affectif féminin.

\section{ESPACES DES QUARTIERS ET SONORITÉS DU QUOTIDIEN}

Ces quartiers sont traversés de rues aux pentes recouvertes d'une insignifiante couche de goudron, érodée peu à peu par les torrents que la pluie y déverse, heure après heure, jour après jour, jusqu'à former des crevasses, des arêtes, des cratères, marques de l'hiver. Plus loin encore, là où les femmes font sécher leur linge sur les fils barbelés du terrain d'en face à vendre, la rue est faite de chemins de terre, qui deviennent une grande mare boueuse pendant la saison des pluies. Pendant la saison sèche c'est la poussière rouge qui s'y accumule. On découvre parfois entre les maisons serrées les unes contre les autres des ruelles improvisées, des passages serrés, improbables, qui se glissent dans les jardins, entre les cours, et constituent la limite entre chaque terrain. Ces maisons forment des lignes, des courbes arc-en-ciel, participant au jeu de la pluie et du soleil. Les murs se colorent d'une semaine à l'autre, repeints de haut en bas avec les couleurs vives de la nouvelle gamme d'une marque bon marché qui attirent l'œil et démarquent ces maisons de celles qui demeurent couleur de brique et de ciment. En se promenant dans ces quartiers, nés au cours de la première moitié du siècle dernier, on se sent entre ville et campagne. Gagnant toujours plus de terrain sur les champs alentour, ils constituent en quelque sorte les marges pionnières de l'urbanisation.

Les parapluies des femmes, les sombrinhas, mettent en mouvement ces chemins, par beau ou mauvais temps. Ils leur permettent de se protéger du soleil ou de la pluie, mais aussi du regard des autres, trop curieux, ainsi que de masquer celui qu'elles portent à leur tour sur ceux et celles qu'elles croisent. Leur ondulation suit le rythme de leurs hanches. Elles sont les actrices principales de ces espaces, dans la durée. En cela, elles en sont une référence incontournable. Au quotidien, ce sont elles qui se retrouvent pour discuter dans les maisons, dans les cuisines et les jardins, mais aussi dans la rue, surtout en fin d'après-midi. Circulant d'une maison à une autre, se rendant visite mutuellement, elles s'échangent des objets, des paroles et des regards qui constituent le liant de leur vie quotidienne auquel les hommes ne participent que de manière indirecte. Ces femmes alimentent ainsi des relations de solidarité au niveau des relations de parenté (mère, sœur, marraine, agregada ${ }^{3}$ entre autres), mais aussi dans le cadre des relations de

${ }^{3}$ On peut dire des agregados et agregadas qu'ils sont membres d'un groupe familial au titre d'amis d'une qualité particulière. Ce sont des proches familiers. Ce statut vient souligner l'importance des relations familiales symboliques dans la société brésilienne. 
voisinage. L'ethnographie que j'ai menée auprès d'elles s'est inscrite peu à peu dans ce tissu relationnel. Pour comprendre leurs présences dans ces quartiers, j'ai accompagné à l'occasion de mes divers séjours leurs trajectoires quotidiennes. Elles sont souvent à l'initiative de déplacements d'un quartier à un autre, de leurs quartiers vers le centre historique de la ville, situé « en bas ", de la ville à la campagne comme elles ont l'habitude de le faire plutôt les dimanches, et de Goiás vers d'autres villes alentour, jusqu'à Goiânia le plus souvent, la capitale de l'État de Goiás. Dans les quartiers, leurs parcours les amènent souvent à passer par la place principale, João Francisco, où l'on trouve plusieurs supermarchés, des magasins de meubles, d'habits, des vendeurs de glace, des bars - botecos, une papeterie, et un peu plus loin, des cybercafés. C'est ici qu'a lieu également le marché du dimanche matin et que se trouve la seule église catholique en dehors du « centre historique », l'église de Santa Rita.

Ainsi, en suivant leurs pas, mes hanches ont adopté leur rythme, et j'ai pu comprendre comment le quotidien de ces quartiers prend forme, et à quel point leurs trajectoires façonnent ces espaces. Ce faisant, il s'agit pour ces femmes de trouver une place légitime dans ces quartiers pour des pratiques et des valeurs issues de la campagne, a roça. Leurs expériences quotidiennes inventent au jour le jour de nouvelles manières de vivre la ville à partir de ces quartiers qui en deviennent des espaces à part entière, ce qui contribue à transformer le paysage de Goiás. L'usage fait ici de la notion de paysage ${ }^{4}$ vient ratifier les propos de Jorge Santiago lorsqu'il écrit que « le paysage est à la fois système d'idées, conception du monde et de pratiques sociales » (2007: 10), et donc $\mathrm{qu}^{\prime}$ " il ne peut exister une perception cohérente du paysage sans la connaissance de la vie sociale qui le remplit, sans la saisie interne des pratiques, des habitudes sociales et culturelles existant chez les individus qui circulent et agissent dans les différents paysages qu'une ville comporte dans sa sédimentation de différents temps vécus » (ibid. : 9).

Les quartiers de la ville de Goiás, comme ceux de nombreuses petites villes du Centre-Ouest brésilien ${ }^{5}$, sont habités par différentes sonorités ${ }^{6}$. On peut entendre au quotidien les annonces faites depuis les haut-parleurs de voitures réservées à cet effet, arpentant les rues des quartiers au pas. Promotions au supermarché, "camions de pastèques", slogans politiques en période électorale sur des airs de musique sertaneja dont les refrains répètent en boucle le numéro du candidat, mais aussi annonces de décès, invitations aux

\footnotetext{
${ }^{4}$ Sur les rapports entre paysage et ethnologie, voir Dubost et Lizet (1995).

${ }^{5} \mathrm{~J}$ 'ai eu l'occasion pendant mes divers séjours de me rendre dans plusieurs autres villes de la région, Itaberaí, São Luis de Montes Belos, Firminopolis, Jussara, Céres, et d'autres que j'ai traversées lors de mes trajets en bus entre Goiânia et Goiás.

${ }^{6}$ Sur les rapports entre le sonore et le social, voir entre autres le travail d'Olivier Féraud consacré au lien entre pratiques vocales et pratiques sociales dans les quartiers Espagnols de Naples, les actions sonores des habitants contribuant à forger une manière propre d'habiter ces espaces (Féraud, 2010).
} 
habitants à se rendre à des débats publics organisés par la municipalité, entre autres. Souvent passées par des voix d'hommes, elles interpellent directement les donas de casa, les maîtresses de maison.

L'architecture locale contribue à créer une ambiance sonore particulière. Les maisons, serrées les unes contre les autres, n'ont pas de revêtement intérieur, et les toitures laissent toujours entrer et sortir une circulation d'air appréciée à l'époque de la sécheresse, qui dure des mois de juillet à octobre, même si les nombreux incendies à cette période remplissent les maisons de cendres noires épaisses qui tombent des jours durant. Quand le vent froid arrive, vers la fin mai, il passe sous les tuiles et traverse les pièces. Les sons aussi s'y glissent, en permanence. D'une chambre à l'autre, d'une maison à l'autre. La limite entre espaces intérieurs et extérieurs est ténue et la présence des autres, visuelle, olfactive et sonore pour ce qui nous intéresse ici, se propage facilement.

Ainsi, les sons se diffusent au dehors, dans des espaces de proximité auxquels ils attribuent une tonalité particulière. En effet, les sons donnent une épaisseur à l'air, le font bouger, vibrer d'une manière spécifique, et l'amènent à conférer aux espaces qu'il traverse une énergie qui leur est propre. Les personnes qui vivent dans ces espaces et qui contribuent à l'émission de ces sons s'en trouvent alors imprégnées. À leur contact, ils sont eux aussi touchés par ces vibrations. Une énergie singulière se dégage alors de la rencontre entre les corps et les sonorités, qui participe à la construction des espaces dans lesquels ils se meuvent ${ }^{7}$. Nous sommes ici tout à fait dans le sillon que de ce François Laplantine appelle une pensée de l'énergie (2005).

Comprendre la manière dont la mise en mouvement des corps de femmes dans les espaces de ces quartiers est liée aux sonorités et plus spécifiquement aux sonorités musicales qui les habitent au quotidien revient à mobiliser la notion d'ambiance sonore dans le sens d'un "phénomène de diffusion du sensible » (Thibaud, $2010: 208$ ) comme l'entendent les chercheurs qui, à la suite de Jean-François Augoyard (1979), réfléchissent en termes d'esthétique des ambiances dans la ville, faisant une lecture sensible de la manière d'habiter les espaces. L'esthétique dont il est question est bien l'Aesthesis, la perception par les sens. Ville à l'épreuve des sens (Thibaud, 2010), ville charnelle (Thomas, 2007, 2010), il s'agit pour ces auteurs de réaliser une écologie sensible, c'est-à-dire de s'intéresser à la manière dont les qualités sensibles d'un environnement modèlent et modulent les déplacements, la gestuelle des corps.

Il est important de mentionner d'autres composantes de l'ambiance sonore de ces quartiers. Les voix tout d'abord, et surtout celles des femmes et des enfants. Elles ne s'élèvent qu'en cas de réprimandes, allant des cris aux pleurs. Les modes d'expression conflictuels entre femmes sont quant à eux presque

\footnotetext{
${ }^{7}$ Les différents aspects de cette thématique ont été l'objet de discussions particularisées avec mon Directeur de thèse, Patrick Deshayes, qui ont suscité de nouvelles réflexions et m'ont ouvert de nouvelles perspectives d'analyse pour la suite de mes recherches.
} 
silencieux. On parle généralement tout bas, la forme narrative privilégiée dans ces cas-là étant les fofocas, les cancans. Il y a également les voix des prières, rezas et ladainhas ${ }^{8}$, plus discrètes que celles des prieuses de neuvaines, rassemblant plusieurs habitants le soir dans les maisons, et que celle du Père Robson dont l'émission matinale sur la chaîne de télévision Rede Viva depuis le lieu de pèlerinage le plus fréquenté de la région, Trindade, où l'on vient vénérer le Divino Espirito Santo, connaît un grand succès auprès des femmes plus âgées. Soulignons que plus récemment, depuis quelques années seulement, on entend passer des voitures dans les rues des quartiers'. Enfin, les sonorités de la télévision avec ses réclames, ses dessins animés, ses telenovelas et ses émissions de divertissement, notamment des chaînes Sbt, Record et Globo, sont presque omniprésentes. Les animateurs parlent fort, souvent sur un fond musical rythmé, festif, avec un enthousiasme débordant caractéristique de cet univers de l'industrie culturelle brésilienne. Les émissions du dimanche ont un succès important, entre autres du fait qu'elles invitent des musiciens, comme dans le programme de la chaîne Sbt, «Domingo Legal».

Ainsi, parmi ces sonorités, c'est à la musique que je donnerai ici une importance particulière. Elle s'écoute depuis la télévision, en DVD, qui ajoute une dimension visuelle à l'expérience sonore, depuis une chaîne hi-fi en CD, ou encore à la radio. La plupart du temps, elle est appréciée avec un volume sonore élevé, qui passe facilement d'une maison à une autre. En parcourant ces quartiers, surtout les dimanches après-midi ou à l'heure de la sieste ${ }^{10}$, on perçoit ainsi aisément plusieurs formes musicales contemporaines émises depuis ces différents appareils présents dans presque tous les salons des maisons, qui plaisent particulièrement aux femmes et aux jeunes filles : le forrón ${ }^{11}$, la musique axé, une tendance musicale urbaine et très commerciale dérivée du samba, la musique brega, sur laquelle nous reviendrons, et surtout, la musique sertaneja. À celles-ci vient s'ajouter le funk carioca, un style musical né dans les années 1980 à Rio de Janeiro qui a pour base rythmique la techno, rythme de discothèque. C'est un genre qui se prête particulièrement bien à l'auto-production, c'est pourquoi l'élaboration des albums, de

${ }^{8}$ Prières quotidiennes de l'univers catholique que l'on fait seul ou à plusieurs le soir, parfois avec un chapelet, près de l'image d'un saint ou d'une sainte.

${ }^{9}$ Leur nombre a considérablement augmenté depuis mon premier séjour en 2006.

${ }^{10}$ C'est d'ailleurs souvent en mettant de la musique que les couples construisent un nid d'intimité, délimité par cette frontière sonore.

${ }^{11}$ Le forró renvoie tout d'abord à des bals populaires de la région du Nordeste brésilien, animés par des rythmes locaux. À partir des années 1950, deux phénomènes ont contribué à son essor dans tout le pays. D'une part, les enregistrements prenant comme thème principal ces bals, et d'autre part, les phénomènes de migration interne du Nordeste vers les grands centres urbains du Brésil, surtout Rio de Janeiro, São Paulo et Brasilia. L'accordéon, le triangle et la zabumba, grand tambour que l'on frappe avec des baguettes, en constituent les trois instruments caractéristiques. Voir le dictionnaire de Musique Populaire Brésilienne Cravo Albin : http://www.dicionariompb.com.br. 
l'enregistrement au montage jusqu'à la diffusion, est réalisée localement, dans les quartiers et pour les quartiers, ce qui va de pair avec une liberté d'expression d'une réalité sociale stigmatisée ${ }^{12}$.

Tous ces genres musicaux sont apparus dans le paysage musical brésilien entre les années 1960 et 2000. Mais après l'essor des mouvements musicaux des années 1970 et 1980, c'est surtout dans les années 1990-2000 que la grande production musicale brésilienne, jusqu'ici concentrée principalement dans les régions de Rio de Janeiro et São Paulo, s'étend à de nouvelles gammes mélodiques, instrumentales et rythmiques, qui reprennent des éléments régionaux de composantes de la société et de la culture brésilienne et en font de nouvelles formes musicales urbaines (Santiago, 2012).

L'État de Goiás n'échappe pas à cette tendance et devient alors la scène principale de l'industrie culturelle de la musique dite sertaneja. Il nous faut nous tourner du côté des ethnomusicologues pour définir cette forme musicale. Son nom renvoie à la musique produite à partir de la fin des années 1920, qui auparavant était désignée par les termes de modas, toadas, cateretês, chulas, batuques, emboladas. Elle est interprétée par des duos constitués la plupart du temps d'hommes aux voix nasales et de fausset, recherchant l'aspect mélodieux plutôt que la puissance (Carvalho, 1995), appelés «Duplas Sertanejas ». Sur cette base, relativement stable, les instruments, les arrangements mélodiques et les rythmes ont changé tout au long du XXe siècle pour adopter des éléments diffusés par l'industrie culturelle brésilienne. Les thèmes des chansons ont toujours traité du quotidien mais se sont consacrés plus particulièrement à l'amour sur un ton autobiographique, dans ce qui, par opposition à la musique sertaneja de raízes, dite « de racines $»^{13}$, est désigné par

${ }^{12}$ Ayant comme sujet principal la violence, le trafic de drogues et l'univers du crime, mais aussi les relations sexuelles entre hommes et femmes, certains compositeurs, acteurs également du narco-trafic, en sont arrivés à être poursuivis par la police entre autres pour les propos tenus dans ces chansons. Soulignons aussi qu'il s'agit d'un genre musical écouté et dansé dans les bals funk des quartiers, des fêtes stigmatisées par les médias qui les associent au crime et à une sexualité débridée, c'est-à-dire à des pratiques déviantes, tant et si bien que, si dans les années 1990 le funk carioca connaît un succès médiatique important qui l'amène à rivaliser avec d'autres rythmes moins controversés, comme la musique sertaneja, à la fin des années 1990 les bals ont été interdits jusqu'à faire l'objet d'une réglementation en 2003 qui contribue notamment à créer de nouveaux lieux considérés comme moins suspects pour ces événements. Concernant ce genre musical, voir les études d'Hermano Vianna (1990, 1988). Pour la définition de ce genre musical, je m'appuie ici en partie sur le dictionnaire de Musique Populaire Brésilienne Cravo Albin. Cf. Infra. Voir également l'article de Sofiane Ailane dans ce volume.

${ }^{13}$ La musique sertaneja de raízes est valorisée notamment par un programme de télévision qui reçoit plusieurs artistes de ce genre musical, Frutos da Terra, diffusé depuis plus de vingt ans sur une chaîne affiliée à la télévision Globo, la télévision Anhanguera, dans les États de Goiás, Tocantins, dans le District Fédéral de Brasília et dans plusieurs villes des États du Pará, Maranhão, Mato Grosso, Mato Grosso do Sul et Minas Gerais. À forte orientation régionaliste, ce programme privilégie les formes d'expression considérées comme propres à la culture goiane, «traditionnelles » et en voie de disparition, alimentant ainsi une nostalgie à leur 
le terme de musique sertaneja romantique. Cette dernière, privilégiant les guitares électriques aux traditionnelles violas ${ }^{14}$, les références urbaines aux rurales, est aujourd'hui et depuis les années 1980 le genre musical le plus produit et écouté au Brésil, s'alimentant d'influences du rock et de la country, que l'on retrouve par ailleurs clairement dans les codes vestimentaires mobilisés. La musique sertaneja met en scène dans le cadre de la ville des sonorités entre autres issues du monde rural, après leur avoir fait subir un processus de modernisation. L'ethnomusicologue Martha de Ulhôa Carvalho écrit dans ce sens : "Les changements de style et le prestige croissant attribués à la musique sertaneja reflètent aussi bien les sentiments personnels envers la vie et les histoires de vie de leurs créateurs, comme ils illustrent l'impact des transformations complexes du Brésil du XXe siècle : migration interne, urbanisation, industrialisation, et modernisation des moyens de communication et de transport. Mon hypothèse est que la musique sertaneja a été utilisée principalement par des migrants comme un moyen de rendre plus facile l'absorption de nouvelles valeurs culturelles - elle devient un moyen d'adaptation des personnes issues de la zone rurale dans la société urbaine » (Carvalho, 1995, traduction libre par mes soins).

Le film de Breno Silveira, 2 filhos de Francisco (2005), qui raconte l'histoire de l'une des Dupla sertaneja les plus célèbres actuellement, Zézé de Camargo \& Luciano, permet de comprendre la transformation de ces musiques dans un contexte d'urbanisation du Centre-Ouest brésilien. Il retrace la trajectoire d'un homme, Francisco, passionné de musique, dont l'ambition est de faire de ses deux fils des musiciens de renom. Travaillant la terre près de Pirenópolis, dans l'intérieur de l'État de Goiás, il est taxé de fou - doido - pour avoir de telles aspirations qui l'amènent à échanger une grande partie de sa récolte contre une guitare et un accordéon qu'il offre à ses aînés âgés d'une dizaine d'années. Il n'hésite pas non plus quelques mois plus tard à changer radicalement de vie en déménageant, lui et sa famille, à Goiânia quand son beau-père lui réclame les terres sur lesquelles il s'était établi : «c'est là-bas où se trouve le futur de ces garçons » dit-il. Nous sommes dans les années 1970, et un impresario repère le duo Camargo \& Camarguinho, leur premier nom de scène, dans la gare routière de la capitale où ils jouent pour tous ceux qui comme eux ont quitté la campagne pour la ville. Après un début de succès qui

égard. On retrouve dans la ville de Goiás cette même tendance de valorisation des traditions goianes, du fait qu'elle est l'objet d'un discours tenu par l'élite locale qui l'associe au berceau de la culture goiane, une stratégie liée à la conquête et au maintien de son titre de patrimoine historique de l'Unesco. Cette valorisation ne concerne pas seulement les expressions musicales locales, mais aussi celles culinaires et poétiques.

${ }^{14}$ Guitare aux cordes doublées, appelée viola caipira ou encore viola sertaneja, utilisée souvent à l'occasion de fêtes populaires dans les milieux ruraux des États de São Paulo, Minas Gerais, Goiás, Mato Grosso et dans une moindre mesure à l'intérieur de Rio de Janeiro. Introduit par les colonisateurs portugais au XVIe siècle, cet instrument est l'un des plus anciens instruments à cordes au Brésil. 
se clôt par un accident de voiture tragique au cours duquel périt le plus jeune des deux musiciens, un second duo se forme dans les années 1990 avec un autre garçon de la famille, Zézé de Camargo \& Luciano. Cette fois-ci, le lieu de conquête de la gloire se déplace et c'est à São Paulo, où le duo se trouve en compétition avec des nouvelles célébrités, que le plus âgé démarche auprès des maisons de disque pour décrocher un contrat. Breno Silveira met à nouveau en scène le père comme l'acteur principal de cette volonté de réussir. Il apporte un premier enregistrement sur K7 à une radio de Goiânia et réussit à créer un auditoire assez important pour que l'une des chansons, É o amor, remporte un record d'audience. Face à ce succès, le producteur accepte de se lancer dans la diffusion de leur premier album. Le film montre ainsi l'ascension sociale de ces garçons, et à travers eux de cette famille, qui ont affronté de multiples difficultés grâce à leur bravoure et à ce qui est perçu comme de l'anti-conformisme de la part de leur père, pour accéder à la reconnaissance nationale d'une musique issue de la terre. Très romantisé, il termine sur une séquence intéressante, la mise en scène du retour à la terre natale cette fois-ci non pas par les acteurs mais par les vrais chanteurs et leurs parents, comme un pèlerinage qui clôt l'histoire sur une note de nostalgie.

Il convient de s'attarder quelque peu sur le regard porté sur ces formes musicales. D'une manière générale au Brésil, les formes artistiques issues des régions rurales et des périphéries urbaines sont méprisées par les classes aisées qui les jugent de mauvais goût, ringardes, simplistes, que ce soit pour leur mélodie, leur rythme ou leurs paroles. En un mot, elles sont considérées comme bregas (Araujo, 1988; Martins, 1990). Le terme brega présente une certaine ambivalence car s'il sert d'une part, et ceci dès les années 1960, à disqualifier plusieurs formes musicales populaires, le plus souvent à forte connotation romantique, comme c'est le cas avec la musique sertaneja, il renvoie par ailleurs à un genre musical particulier. Il recouvre donc plusieurs sens qui sont liés entre eux, du fait qu'il peut s'agir d'un adjectif qualificatif imposé de l'extérieur aux classes populaires jugées pauvres culturellement telle ou telle musique est brega, mais aussi d'un style musical relativement récent qui mobilise ce vocable comme pour retourner le stigmate qui lui était jusqu'ici rattaché, dans le sens goffmanien du terme (Goffman, 1975), dans l'objectif de se l'approprier et ainsi de s'auto-désigner. C'est ainsi que naît dans les années 1990 la "musique brega » portée par des artistes de Belém, dans l'État du Pará, comme pour défier les normes esthétiques des classes aisées, et dans son sillon, la "musique brega calypso », qui connaît un succès important également dans les quartiers de Goiás ${ }^{15}$.

Faut-il voir dans l'existence d'un tel préjugé l'une des raisons pour lesquelles les anthropologues ne s'intéressent que trop peu à la musique sertaneja ? Dans quelle mesure ce silence à leur égard contribue-t-il au défaut

${ }^{15}$ Le terme calypso renvoie à la musique de carnaval issue de la Caraïbe, notamment de Trinidad et Tobago, mais adopte une tonalité plus romantique au nord du Brésil. 
de reconnaissance qui les touche ? Comme l'écrit Martha de Ulhôa Carvalho, qui s'est prêtée à l'exercice de dégager l'esthétique propre à cette musique à partir d'une ethnographie à Uberlândia dans l'État de Minas Gerais, "la plupart des travaux, en nombre assez réduit d'ailleurs, qui traitent de la musique sertaneja, interprètent sa signification du point de vue de sa production, c'est-à-dire en l'approchant par le biais de ses aspects industriels, par opposition à ses origines artisanales » (Carvalho, 1995, traduction libre par mes soins). Remarquons que, même si la plupart des travaux auxquels l'auteur renvoie ont été réalisés avant les années $1990^{16}$, date à laquelle cette forme musicale a connu une transformation importante, il n'est pas difficile de saisir ici l'enjeu de taille que se proposent de relever ceux qui choisissent d'étudier ces formes musicales. Il s'agit en effet d'éviter deux biais qui témoignent des préjugés que le chercheur a du mal à expliciter vis-à-vis de son sujet de recherche, le mépris et la nostalgie, et ainsi d'éviter l'appauvrissement d'une pensée binaire qui oppose la ville et la campagne, la tradition et la modernité, les classes populaires aux classes aisées. Ces préjugés, qu'ils soient négatifs ou positifs, contribuent à alimenter l'illusion d'une imperméabilité entre les formes expressives populaires de la société brésilienne et les autres.

Il s'agit ici au contraire de mettre en avant les rapports entre les différentes réalités sociales, esthétiques et sensibles que semblent opérer la musique sertaneja aujourd'hui. Au-delà de l'importance de sortir des préjugés, prêter l'oreille à ces sonorités spécifiques et faire une analyse de ce qu'elles produisent en termes de corporéités présente un intérêt majeur, du fait qu'il s'agit des formes d'expressivité privilégiées par les femmes avec lesquelles j'ai cohabité sur le terrain. Si ces sonorités musicales sont minorées ailleurs, elles sont présentes partout dans les quartiers de Goiás.

\section{MUSIQUES ET CHORÉGRAPHIES DE LA SÉDUCTION}

Comment les femmes de ces quartiers éprouvent ces ambiances musicales ? De ce que j'ai pu observer sur le terrain avec elles, les chansons interprétées par les artistes de musique sertaneja tiennent une place particulière dans leur univers affectif. En outre, ces écoutes quotidiennes suscitent de leur part des réponses corporelles et participent ainsi aux modalités de déplacement et à la gestuelle de leurs corps ${ }^{17}$, qui n'est pas sans lien avec les rapports qu'elles entretiennent avec les hommes, notamment quand elles sortent danser. Ainsi,

\footnotetext{
${ }^{16}$ Elle se réfère entre autres aux ouvrages de Waldenyr Caldas $(1979,1987)$ et à ceux de José de Souza Martins $(1974,1975)$.

${ }^{17}$ La sociologue britannique Tia De Nora a orienté nombre de ses travaux en Grande-Bretagne sur la manière dont le corps se meut au contact de la musique, parlant de cette dernière comme d'un «ingrédient actif » sur les corps, et s'intéressant à la manière par laquelle elle «entre en action» dans différents contextes sociaux, comme par exemple dans le cadre de cours d'aérobic ou encore dans des magasins de vente au détail (De Nora, 2001).
} 
je fais le constat que, provoquées entre autres par ces sonorités, les expériences sensibles que les femmes vivent dans ces quartiers au quotidien, mais aussi en situation festive, participent à l'élaboration d'un univers féminin singulier.

Il convient de s'intéresser à ce que les chansons, en tant que forme narrative spécifique, racontent. La plupart de celles qui composent les répertoires de sertanejo romântico ont pour thème principal, comme le genre musical auquel elles appartiennent l'indique, des histoires d'amour ou de chagrin d'amour. Un point important à souligner ici concerne les interprètes de ces chansons. Il s'agit surtout d'hommes en duo ou en solo, dont les voix douces et plutôt aiguës sont appréciées des auditrices. À ce sujet, remarquons qu'on voit apparaître beaucoup plus récemment, à partir des années 2000, une nouvelle configuration avec des chanteurs en solo. Ce ne sont plus les duos qui prédominent, et l'on peut d'ailleurs noter un changement léger de registre vocal car la voix de fausset tend à disparaître, comme chez Léo Magalhães. Son titre Locutor (Ao Vivo em Goiânia, 2009) était l'une des chansons les plus écoutées par les femmes lors de mon dernier séjour à Goiás en 2010. En voici les paroles :

« Je suis sur mon portable

Je te parle depuis un bar

Où j'ai tout bu pour pouvoir téléphoner

J'ai perdu un grand amour

Je ne sais pas quoi faire

Ami locuteur, je t'appelle pour te dire

Envoie-lui un message et un baiser de ma part

Je sais qu'elle ne perd aucun de tes programmes

Elle n'ouvre pas la porte et n'a pas de portable

Alors il n'y a qu'une solution pour qu'elle m'écoute

Allez locuteur dis-lui que je suis complètement amoureux, fou d'amour

Dis-lui s'il te plaît, dis à l'antenne, je ne sais pas vivre sans elle

Qu'il y a un mec ici, à qui elle manque, plein de désirs

Dis-lui locuteur, je ne veux qu'un baiser de plus ${ }^{18} »$.

Une analyse rapide de ce texte met en évidence l'intérêt qu'il peut représenter pour celles qui écoutent cette chanson. Elle met en scène l'histoire d'un chagrin d'amour ou d'un amour non partagé à partir d'un univers considéré comme masculin, le bar, et de plusieurs références à des outils

${ }^{18}$ Traduction libre par mes soins de :

"Tô no celular / Falando de um bar / Bebi todas pra poder ligar Perdi um grande amor / Não sei o que fazer / Amigo, locutor liguei pra te dizer

Manda um recado e um beijo meu / Sei que ela não perde um programa seu / Ela não abre a porta e não tem celular/ Então só tem um jeito dela me escutar

Vai locutor / Diz que eu tô / Completamente apaixonado, louco de amor/ Diga por favor, fala aí no ar, não sei viver sem ela/ Que tem um cara aqui, com saudade dela, cheio de desejos / Diga a ela locutor só quero mais um beijo". 
technologiques, la radio et le téléphone portable, dont l'utilisation est devenue ordinaire dans le quotidien des quartiers de Goiás. Le plus intéressant consiste dans le fait de prendre à parti une tierce personne pour exprimer ses sentiments, personne qui devient le personnage central du récit, en l'occurrence ici, le locuteur. Cette démarche, adoptée pour résoudre les questions affectives quand tout semble aller à l'encontre d'une relation entre un homme et une femme, est assez commune dans l'univers quotidien des auditrices. On imagine que pour elles, le locuteur peut tout aussi bien être l'auditeur, ou plutôt l'auditrice, qui est en posture de se sentir directement impliquée dans cette histoire. La construction narrative de cette chanson laisse une marge de liberté à celle qui écoute de se mettre à la place, ou pas, du locuteur. Il peut donc s'agir de l'homme malheureux qui transmet un message à la femme aimée par l'intermédiaire du téléphone puis de la radio, s'adressant directement au locuteur, ou du chanteur qui, dans le même but, mobilise l'intermédiaire d'autres femmes qui l'écoutent. En effet, n'oublions pas que la plupart des chansons de la musique sertaneja sont d'inspiration autobiographique.

Comprendre pourquoi les femmes aiment écouter ces histoires d'amour interprétées musicalement par des hommes qui s'adressent à elles, indirectement ou directement, exige de s'attarder quelque peu sur la place que prend cette expérience de l'écoute dans leurs univers affectifs. En effet, comme le remarque si justement Jorge Santiago, la chanson peut être un "véhicule utilisé pour exprimer publiquement des sentiments comme l'amour, la honte, la jalousie, la nostalgie, le mépris » (2009: 134), et peut générer « un climat de liberté d'expression pour des hommes et des femmes des couches populaires, associant le passé et le présent, la passion et la douleur, la plaisanterie et le travail » (ibid. : 135). On parle de choses qui ne se disent pas ailleurs, ni autrement.

$\mathrm{Qu}^{\prime}$ un homme exprime ainsi ses sentiments envers une femme, bien que de manière indirecte, est certainement ce qui octroie autant de succès, dans un public constitué avant tout de femmes, à la chanson de Léo Magalhães. En effet, c'est un sujet dont on ne parle pas entre hommes et femmes, surtout parce qu'on attend d'un homme qu'il ne manifeste pas ses sentiments, faute de quoi, aux yeux des autres et surtout des autres hommes, sa virilité pourrait $\mathrm{s}^{\prime}$ en trouver menacée et il se verrait taxé de "boiola ${ }^{19}$, c'est-à-dire $\mathrm{d}^{\prime}$ homosexuel. Comme les femmes n'ont pas accès directement à leur univers affectif, elles se font un avis de la manière dont ils envisagent leurs rapports avec elles, à partir de ce qu'ils se disent entre eux. J'ai souvent pu écouter de mes interlocutrices, à l'occasion de discussions entre femmes dans les cuisines, ou encore au salon de beauté, ce qu'elles imaginaient que les hommes pouvaient dire à leur sujet. Ces femmes sont pour la plupart mères et

\footnotetext{
${ }^{19}$ Expression locale à caractère péjoratif pour désigner les hommes homosexuels.
} 
célibataires ${ }^{20}$, et celles qui sont mariées souffrent fréquemment des infidélités de leurs maris, d'autant plus qu'elles finissent souvent par l'apprendre très vite. Selon elles, ce qui pousse les hommes à s'intéresser aux femmes, ce sont les relations sexuelles. "Les hommes sont des bons à rien, la chaire est faible », ai-je entendu à plusieurs reprises, ou encore « les hommes sont des animaux, ils aiment ça, tout est sexe pour eux ». Autrement dit, ils ne résisteraient pas à la tentation sexuelle que constituerait pour eux le corps d'une femme.

Pour revenir aux chansons de musique sertaneja, elles apportent toute une rhétorique sentimentale qui vient répondre à ce que ces femmes attendent idéalement de la part d'un homme, ce qu'elles aimeraient écouter de leur bouche, quand leur quotidien les confronte souvent à des silences, à du nondit qui alimente chez elles l'idée d'une absence de sentiments de leur part à leur égard. C'est dans ce sens que mon hôte me demandait souvent lors de mes venues, non sans humour, de lui apporter dans mes valises « un homme avec un $\mathrm{H}$ majuscule » pour reprendre ses termes ${ }^{21}$, car, toujours selon elle, il n'y avait à Goiás pas d'hommes qui valaient le coup, seulement des homosexuels ou «des hommes avec un $\mathrm{H}$ minuscule » qui ne servaient qu'à faire souffrir. Si les hommes ne doivent pas montrer leurs sentiments, les artistes bénéficient comme d'un droit particulier en la matière, car ce rôle semble leur être réservé sans qu'ils soient pour autant considérés comme moins virils. Ils viennent ainsi combler un manque d'expression et d'écoute dans les rapports que les femmes entretiennent avec les hommes. C'est dans cette mesure que ces esthétiques sonores musicales et leur apport narratif participent de l'élaboration d'un univers affectif féminin.

L'histoire de Divina est particulièrement intéressante à ce sujet. Mère de deux adolescents, elle a fini par abandonner l'idée de construire une relation durable et assumée avec un homme. D'abord parce qu'il lui était difficile $\mathrm{d}^{\prime}$ imaginer vivre avec ses enfants sous le même toit qu'un autre après sa séparation, du moins pas avant que ces derniers soient partis faire leur vie ailleurs $^{22}$. Aujourd'hui, ses enfants étant sur le point de quitter la maison et de s'assumer financièrement, elle envisage cette possibilité, pressée que sa fille, fiancée depuis deux ans, se «case $»^{23}$. Alors que dans l'expression «mère

${ }^{20}$ Il ne s'agit pas ici d'enfermer les femmes dans cette condition de «mère célibataire ", mais de la signaler en tant qu'élément à ne pas négliger dans l'appréhension de la réalité sociale locale. Cette condition concernant d'ailleurs de nombreuses femmes rencontrées sur le terrain est l'objet d'une réflexion approfondie dans le cadre de mes travaux en cours.

${ }^{21}$ "Ser homem com $\mathrm{H}$ », «être un homme avec un $\mathrm{H}$ », était jusqu'à récemment une expression commune au Brésil pour désigner la masculinité.

${ }^{22}$ Cette posture, fréquente chez les femmes dans la même situation, est due en grande partie à une peur des violences sexuelles qui sont comprises comme une conséquence presque naturelle de la cohabitation entre les jeunes filles et leur beau-père. Car si « la chair est faible", on considère qu'elle l'est d'autant plus face à un corps féminin jeune, qui plus est, vierge.

${ }^{23}$ Le terme casar signifie l'engagement que prend un couple quand il décide de vivre ensemble, que ce soit par le mariage ou par le fait de vivre sous le même toît, dans la même maison, a casa. On pourrait donc le traduire par le verbe «se marier », mais cela simplifierait 
célibataire ", la mère prédominait sur la femme célibataire, désormais elle sent que pour elle, la tendance s'inverse, car elle a fini d'élever ses enfants. Lors de mon dernier séjour, elle $\mathrm{m}^{\prime} \mathrm{a}$ confié avoir depuis peu des relations avec plusieurs hommes, ses ficantes comme elle les appelle, qu'elle retrouve de temps en temps dans les motels situés à la sortie de la ville ${ }^{24}$. Le terme ficar, "rester », est assez récent au Brésil et renvoie au fait d'avoir une relation intime passagère avec quelqu'un. Divina a trop souffert de désillusions amoureuses et a donc décidé de se désengager affectivement, sans pour autant renoncer au plaisir sexuel que peut lui procurer une relation avec un homme. En même temps, elle continue à alimenter certains éléments de sa vie affective en écoutant quotidiennement des chansons comme celle présentée ci-dessus.

Les chansons interprétées par les artistes de musique brega calypso et funk carioca sont quant à elles beaucoup plus sexualisées, et prennent un ton humoristique, léger et même graveleux. Lors de l'un de mes séjours auprès de ces femmes, la fille de mon amie chez qui je logeais alors, âgée de 18 ans, faisait souvent allusion, avec un air malicieux, à une chanson au titre évocateur, A piriquita, terme féminisé de periquito, la perruche, pour désigner le sexe féminin. Elle l'avait enregistrée sur son portable et me la fit écouter un jour, en la présence de sa mère, juste après le départ de sa grand-mère. En entendant la phrase principale, répétée de nombreuses fois par une voix de femme, "Qui va vouloir ma piriquita, ma piriquita, ma piriquita / Qui va manger ma piriquita... $\aleph^{25}$, et en constatant ma réaction de surprise et d'amusement, elles éclatèrent de rire. Au Brésil, l'acte de manger correspond au fait de se nourrir, mais aussi à l'acte sexuel. La question de la nourriture, $a$ comida, fait partie de tout un univers de plaisirs et de séduction, qui permet de comprendre la manière dont la sexualité est conçue. Roberto Da Matta écrit à ce sujet : « la comida, comme la femme (ou l'homme dans certaines situations), disparaît dans le mangeur (comedor) - ou gourmand - comilão. C'est la base de la métaphore en ce qui concerne le sexe, qui indique que le mangé est totalement embrassé par le mangeur $\gg^{26}$. On pouvait écouter dans les maisons à peu près à cette période une autre chanson sur le même registre, Saia $e$ bicicletinha (2009) du groupe Banda Kaçamba, que j'ai entendue pour ma part dans la bouche d'une petite fille de six ans, suscitant les rires de sa mère. En voici les paroles :

le sens qui lui est attribué localement. À défaut d'un terme plus adéquat, j'opte ici pour la traduction l'expression française "se caser», qui renvoie au fait d'assumer une relation durable à deux aux yeux des autres et pour soi.

${ }^{24}$ Les motels sont des hôtels de voyageurs situés à l'entrée des villes ou à proximité des axes routiers qui sont devenus des lieux destinés à répondre à une quête de privacité des couples.

${ }^{25}$ Traduction libre par mes soins de la chanson interprétée par Lene Silva et le groupe Balança Nenem, «Quem vai querer a minha piriquita / Quem vai comer a minha piriquita...».

${ }^{26}$ Concernant cette question, voir également Richard Parker (1991). 
«Elle sort en jupe à bicyclette, une main sur le guidon et l'autre qui couvre sa culotte

Ca me fait frissonner quand elle pédale, mais il y a une main devant qui gêne tout le temps

Je crois qu'elle a peur que la perruche s'envole, c'est pourquoi elle continue de couvrir

Mets ta jupe et viens dans la rue avec ta bicyclette, je veux voir la couleur de ta culotte

Elle sort en jupe à bicyclette, une main sur le guidon et l'autre qui couvre sa culotte ${ }^{27}{ }^{27}$

Ces musiques, qui parlent d'une manière générale des rapports entre hommes et femmes, peuvent être distinguées entre musiques romantiques et musiques sexualisées. Une distinction thématique qui vient marquer une séparation entre les sentiments amoureux et les pratiques sexuelles, et qui se trouve en rapport avec des contextes sociaux d'écoute différents.

Comment et dans quel contexte s'écoutent ces musiques au quotidien ? L'écoute musicale est une pratique sociale qui met en rapport la musique avec un ensemble d'autres éléments qui viennent l'accompagner, comme la danse, le chant, le travail, entre autres. C'est, pour reprendre les mots de Jorge Santiago, " une attitude qui dépasse la simple audition » (Santiago, 1998 : 234). En effet, tel qu'il le signale et comme j'ai aussi pu le constater, il est commun au Brésil d'écouter la musique au quotidien en y associant une réponse par le corps, et ainsi de 1' " accompagner ». Dans ces quartiers de Goiás, la musique comme élément prédominant de l'ambiance sonore suscite une gestuelle corporelle spécifique chez les femmes, des mouvements du corps singuliers sur lesquels il faut maintenant s'attarder un peu.

Tout d'abord, j'ai pu observer différents contextes d'écoute à l'intérieur même des maisons. Il existe une écoute que l'on peut appeler solitaire, non pas dans le sens où la personne se trouve seule dans la maison à ce moment-là, mais dans celui où la musique écoutée n'est pas l'objet d'un échange, que ce soit narratif ou corporel. Elle se produit pendant les travaux domestiques, des moments où l'on cuisine, mais surtout à l'occasion des pratiques de nettoyage, de la maison et des habits, autour du tanque ${ }^{28}$ principalement. La musique donne aux corps des travailleuses un rythme. Elle rythme le mouvement des

${ }^{27}$ Traduction libre par mes soins : « Ela sai de saia de bicicletinha uma mão vai no guidon e a outra tapando a calcinha $\{$ bis $\}$ / Da um arrepio quando ela sai pedalando mais tem uma mão na frente que tá sempre atrapalhando / Acho que ela tem medo do piriquito voar por isso que ela não para de tampar \{bis\}/ Eu não aguento mais essa situação vamos liberar geral vamos tirar essa mão / Bota a saia e vem pra rua com sua bicicletinha eu quero ver a cor da sua calcinha / Ela sai de saia de bicicletinha uma mão vai no guidon e a outra tapando a calcinha $\{$ bis $\} »$.

${ }^{28}$ Le tanque est constitué de plusieurs éviers en ciment, dont un sans robinet avec une évacuation d'eau, qui se trouvent souvent à proximité de la cuisine, dans un espace couvert appelé l'área ou ailleurs sur le terrain de la maison. 
bras qui frottent le tissu contre la pierre, qui versent l'eau sur le sol en béton des pièces de la maison, qui nettoient dans les moindres recoins, sous tous les meubles, la poussière accumulée. Leur écoute, qui est souvent chantée et dans une certaine mesure, dansée, remplace les chants de travail comme les cantos de eito rythmaient autrefois le travail difficile des esclaves aux champs.

Cette écoute solitaire contraste avec une écoute collective, qui relève plutôt de moments de détente et d'amusement entre femmes, des moments de brincadeira, déclenchés à l'initiative des jeunes filles, ou des mères quand leurs filles sont encore très jeunes. Là, les mouvements du corps qui accompagnent la musique sont clairement de l'ordre de la danse, de chorégraphies qui se diffusent sur des modèles précis par le biais de DVD musicaux enregistrés par les artistes, achetés sur le marché et copiés. C'est l'occasion de se divertir autour de ce dont chacune est capable ou non de faire avec son corps pour suivre la musique. À plusieurs reprises, il nous est arrivé de nous retrouver, avec des jeunes filles et leurs mères, dans le salon de la maison à nous défier mutuellement pour danser sur du funk carioca, du hip-hop ou de la musique brega ou axé. "Vous êtes déchaînées, vous avez le feu à la casserole !" nous lançât une fois mon hôte, à sa fille et à moi, pour nous signifier que pour bouger autant sur ce type de musique, nous devions être bien excitées. Une autre interlocutrice avait aussi l'habitude de mettre de la musique pour danser avec sa fille de six ans. Un jour, alors que sa nièce âgée de quelques années de plus était là aussi, nous dansions toutes dans le salon sur la musique brega calpyso d'un groupe à succès, la Banda Djavu ${ }^{29}$. Les fillettes cherchaient à reproduire la chorégraphie des danseuses du groupe, qu'elles avaient vue à la télévision ; pour cela, elles avaient détaché leurs longs cheveux et les rejetaient en arrière avec un mouvement circulaire de la tête, dansant tantôt ensembles, tantôt chacune de son côté.

Des remarques ponctuent ces moments de rigolade entre femmes qui réunissent plusieurs générations dans un même espace autour de la mise en mouvement du corps. Car il s'agit d'un moment pendant lequel se joue l'apprentissage $d^{\prime}$ une mise en scène corporelle, dans un sens nettement sexualisé. Cette dynamique peut être mise en rapport avec une autre, similaire, que Sarah Baker (2010) rapporte dans son étude menée auprès de pré-adolescentes en Australie, soulignant l'importance de cet apprentissage chez les jeunes filles autour de l'écoute de certaines musiques. Elle montre comment leurs mises en scène corporelles correspondent alors à une exploration des limites de la séduction, partie prenante de la construction de soi. À Goiás, les femmes plus âgées disent souvent de celles qui ont accédé à cette étape qu'elles sont "un enfant dans un corps de femme ». Il s'agit donc de gérer l'éveil de la sexualité à partir de ce que ces jeunes filles font, ou pas,

\footnotetext{
${ }^{29}$ La Banda Djavu est un groupe formé à la fin des années 2000 dans l'État du Pará, dont le succès important dans le nord du pays gagne peu à peu d'autres régions, comme le CentreOuest.
} 
de leur corps. Elles doivent apprendre à l'adopter, c'est-à-dire apprendre à la fois qu'elles peuvent paraître séduisantes et à se protéger des hommes. L'acquisition de la juste distance, être séduisante et désirable sans être prise pour une "fille facile», passe par ces moments de plaisanterie, de jeu autour du corps mis en mouvement pour élaborer de véritables chorégraphies de la séduction $^{30}$.

D'autres situations autour de ces chorégraphies particulières permettent de comprendre l'importance du rôle de ces plaisanteries en ce qui concerne la sexualisation des corps de femmes. Présentons l'une d'entre elles, la «danse de la bouteille», une chorégraphie qui accompagne la chanson du groupe d'axé É o Tchan, Na boquinha da Garrafa (2002), mais est utilisée également pour d'autres rythmes. Les paroles de cette chanson guident les pas à adopter :

«Au samba elle m'a dit qu'elle se frottait

Au samba je l'ai déjà vue se déhancher

Au samba elle aime le frottis-frottas

Elle m'a échangée contre une bouteille

Elle n'a pas supporté et elle est partie se frotter

Frotte-toi au goulot de la bouteille, oui au goulot de la bouteille

Descends sur le goulot de la bouteille, oui au goulot de la bouteille

Descends encore, descends encore un peu, descends encore, descends doucement

Ressors du goulot de la bouteille, oui du goulot de la bouteille

Remonte du goulot de la bouteille, oui du goulot de la bouteille

Monte encore, encore un peu, monte encore, monte doucement

Oui, elle aime le frottis-frottas et dans l'élan du samba elle ne pense qu'à se frotter

Elle aime le frottis-frottas, tu as vu le goulot de la bouteille

Elle n'a pas supporté et elle est partie se frotter

Frotte-toi au goulot de la bouteille, oui au goulot de la bouteille

Monte et descends sur le goulot de la bouteille, oui sur le goulot de la bouteille $»^{31}$.

30 En dialoguant avec les analyses de Cécile Dauphin et Arlette Farge, je fais de ces chorégraphies et des différentes modalités de "ritualisation du désir et de l'approche amoureuse » (Dauphin et Farge, 2001 : 10) l'objet d'analyses particulières dans le cadre de mes travaux. Pour ce qui est d'une réflexion plus générale sur la notion de séduction, voir également Jean Baudrillard (1979).

${ }^{31}$ « No samba ela me disse que rala / No samba eu já vi ela quebrar / No samba ela gosta do rala, rala / Me trocou pela garrafa / Não agüentou e foi ralar

Vai ralando na boquinha da garrafa / É na boca da garrafa / Vai descendo na boquinha da garrafa /É na boca da garrafa / Desce mais, desce mais um pouquinho /Desce mais, desce devagarinho

Vai saindo da boquinha da garrafa / É da boca da garrafa /Vai subindo na boquinha da garrafa /É da boca da garrafa / Sobe mais, sobe mais um pouquinho /Sobe mais, sobe devagarinho

Sim, ela gosta do rala, rala e no embalo do samba / Ela só pensa em ralar /Ela gosta do rala, rala, viu a boca da garrafa / Não agüentou e foi ralar

Vai ralando na boquinha da garrafa /É na boca da garrafa / Sobe e desce na boquinha da garrafa /É na boca da garrafa » (Traduction libre par mes soins). 
Il s'agit de danser les jambes écartées au-dessus d'une bouteille avec un mouvement qui suggère l'acte sexuel. Le terme «boca», sur lequel l'auteur a vraisemblablement joué, contient une ambiguiité. La bouche de la bouteille peut renvoyer par association au sexe de la danseuse, car ce terme désigne aussi localement le sexe d'une femme. Danser au dessus de la bouche de la bouteille - que j'ai traduit ici par goulot -, c'est faire danser sa propre bouche, en l'occurrence, faire danser son sexe.

Une autre interlocutrice, Rosimeire, me dit un jour de sa fille qui s'essayait à une chorégraphie de ce type : "regarde comme elle rebola!». Le rebolado correspond à un mouvement circulaire des fesses et des jambes, d'avant en arrière mais aussi à la verticale, qui amène le corps à tourner sur lui-même et donc potentiellement autour d'un autre corps, de multiples façons. On peut le rapprocher de la ginga que François Laplantine considère avant tout comme un mouvement féminin. «On parle du gingado des jeunes filles ou des jeunes femmes en prêtant une attention particulière au mouvement de balança (balancement) de leurs hanches » écrit-il (Laplantine, 2005 : 20). Rebolar c'est danser, mais c'est aussi un mouvement qui renvoie à l'acte sexuel. Ce mouvement corporel est donc l'objet de jugements de valeur. Dans ce sens, François Laplantine écrit encore au sujet du gingado : «il ne manque pas d'apparaître comme une façon de se comporter avec son corps quelque peu suspecte, voire immorale, aux yeux d'une partie de ceux qui appartiennent à la classe moyenne, et plus encore à la bourgeoisie. Il est perçu comme un relâchement tant physique que psychologique » (ibid. : 22).

On rejoint ici la question mentionnée plus haut du regard porté sur ces genres musicaux, qui ne peuvent être dissociés des pratiques corporelles les accompagnant, lesquelles ne sont pourtant pas perçues par ces femmes comme quelque chose de simplement vulgaire. Là réside toute l'ambiguïté. Il semble plutôt qu'elles jouent par leurs corps avec la limite de l'acceptable, quand la séduction frôle la vulgarité mais ne s'y circonscrit pas. Ce qui pourrait être taxé de la sorte est immédiatement objet de dérision. C'est surtout l'occasion pour les femmes d'aborder leur sexualité sur le mode de la plaisanterie à partir d'un moment de déploiement d'une forme de sociabilité féminine spécifique aux relations entre femmes proches, qui entretiennent entre elles des relations de parenté et en particulier des relations de transmission intergénérationnelle ${ }^{32}$. Les brincadeiras sont mobilisées pour se dévoiler, provoquer, s'esquiver, parler de façon détournée de sa vie sexuelle, de ses désirs, des attentes qu'on a en tant que femme envers les hommes. Le rire permet d'élaborer une moralité malgré tout présente, mais qui se trouve détournée dans ce cadre. On comprend mieux alors la situation relatée précédemment autour de la chanson A piriquita. Un autre jour, la jeune femme fit écouter cette musique à un invité de la famille, quand, surpris, ce dernier

\footnotetext{
${ }^{32}$ À ce sujet, je me permets de renvoyer à l'un de mes travaux (Rougeon, 2012).
} 
lui demandât si sa mère l'autorisait à entendre ce genre de propos. Elle répondit : «Oui ! Ma grand-mère elle, elle trouve ça drôle. Mais si c'était pour danser, je ne crois pas qu'elle me laisserait faire. Je n'ose pas non plus, c'est dépravé...».

\section{SORTIR DANSER}

Les situations relatées ici permettent de comprendre comment l'écoute que ces femmes réservent à ces musiques s'inscrit dans leur vie affective et sexuelle avec les hommes et participe ainsi à la construction corporelle de leur féminité. Ces quelques éléments ethnographiques me conduisent maintenant à articuler à nouveau les espaces intérieurs des maisons aux espaces extérieurs, à partir des lieux où l'on sort danser le week-end. Danser entre femmes pour plaisanter, c'est aussi une manière de se préparer pour danser les jours de fête au regard des hommes et pour séduire.

Ces sorties entre femmes sont précédées de préparatifs qui relèvent de la présentation de soi, de la mise en scène des corps, auxquels sont réservés un temps spécifique, souvent le samedi, et des lieux particuliers, à la maison, chez une proche ou au salon de beauté - salão - d'une connaissance. Les cheveux sont lavés, on leur passe de la crème, on leur fait des masques, des bigoudis pour les hydrater, on les lisse au fer - chapinha, ou on leur fait une escova au sèche-cheveux pour donner une autre apparence à ceux qui, rebelles, persistent à vouloir friser. Il est d'ailleurs intéressant de souligner que les cheveux frisés sont communément appelés "mauvais cheveux », cabelo ruim. Mains et pieds reçoivent autant d'attention. Ils trempent dans de l'eau savonneuse, sont frottés, dessous, dessus, sous les ongles, entre les doigts, puis hydratés, les peaux mortes coupées, les ongles limés et peints, parfois de plusieurs vernis différents superposés pour atteindre la couleur désirée, un choix qui varie en fonction de plusieurs critères, dont la couleur de la peau. Ces soins portés au corps constituent le préambule à la présentation publique de soi devant les hommes mais aussi devant les autres femmes. Elles s'ornent ensuite de bijoux brillants, en toc doré, moulent leur poitrine dans des hauts serrés et décolletés, leurs jambes dans des pantalons bien ajustés. Le rouge des lèvres et des ongles est profond et le brillant de leurs cheveux tirés en arrière retombe sur leur nuque. Une fois prêtes, elles se réunissent pour sortir ensemble. Il faut aller chercher unetelle qui habite à l'autre bout du quartier, attendre qu'elle ait fini de se coiffer, l'aider éventuellement à choisir une robe plutôt qu'une autre, l'assortir à une paire de chaussures...

Sortir danser est une pratique qui demande à la fois une préparation du corps, mais aussi une préparation sociale qui engage une forme singulière de sociabilité féminine, la solidarité. Toutefois, il convient de souligner que l'esthétique que ces femmes élaborent à partir de leurs corps alimente également des jeux de pouvoir entre femmes basés sur le sentiment de l'envie, 
inveja, provoqué par des échanges de regards. En effet, les attributs physiques des unes sont convoités, admirés par les autres. Les soins sont portés au corps en vue de la présentation publique de soi devant les hommes, mais aussi devant les autres femmes, et participent dans ce sens à éveiller des relations de rivalité. Analysant le rôle de la beauté dans l'établissement des institutions humaines, et plus précisément, la manière par laquelle cette dernière, inégalement répartie, est l'objet d'une régulation sociale, Pierre-Joseph Laurent (2010) propose de comprendre les relations de rivalité entre femmes comme l'un des processus de cette régulation, présent dans plusieurs sociétés (Grèce Antique, Na de Chine, Mossi du Burkina-Faso, entre autres). L'auteur écrit dans ce sens, «La beauté émanerait [alors] du sentiment de jalousie ou de rivalité que [les femmes] entretiennent entre elles, en raison d'un différentiel de beauté et de ses conséquences dans leurs quêtes pour attirer un homme, pour l'avoir pour elles et ensuite le garder.» (Laurent, $2010: 57$ ).

D'abord confrontée à l'idée selon laquelle Goiás est uma cidade parada, une ville arrêtée, sans mouvement, une phrase que j'ai entendue d'innombrables fois, avec le temps j'ai découvert une vie nocturne propre aux habitants de ces quartiers $^{33}$. La première image qu'on

m'a donnée des lieux où l'on sort danser se voulait dissuasive :

«Le Sayog n'est pas un lieu pour une femme mariée » me dit un ami de la famille chez qui j'étais accueillie, «c'est une porcherie». Et au Morro do macaco molhado poursuivit-il, il n'y a que « des hommes mariés et des homosexuels. Peut-être qu'il y a même une chambre pour d'autres choses... Là-bas ça boit, ça danse, on s'embrasse et un peu plus. C'est pour finir la nuit, quand les gens sont bien saouls ».

Ainsi, on ne dit pas à n'importe qui que l'on sort danser et où, car la fréquentation de ces lieux est associée aux rencontres amoureuses entre hommes et femmes. Pour des femmes, ce serait laisser penser que l'on va rejoindre des hommes pour draguer, pour namorar, c'est-à-dire avoir une relation amoureuse, ou encore pour ficar, se donner du plaisir le temps d'une soirée. Précisons que l'engagement dans ce type de relations varie si l'on est un homme ou une femme, dans le sens où c'est socialement acceptable pour eux, même valorisé et proclamé, alors que cela doit se produire pour elles sur le mode du sous-entendu, de la brincadeira. D'ailleurs, toutes les femmes ne peuvent pas se permettre de sortir danser. Les jeunes filles qui se rendent à ces fêtes font fréquemment le mur, avec la complicité d'une cousine plus âgée ou

\footnotetext{
${ }^{33}$ Les lieux où l'on sort danser ne sont pas accessibles au premier abord à ceux qui, comme moi, viennent de « dehors ». Les nuits goianes pour les touristes venus de Brasília, Salvador ou d'autres pays sont celles du centre historique, mouvementées toute l'année par plusieurs événements culturels, comme le Fica - Festival International de Cinéma de l'Environnement, ou la Semaine de la Conscience Noire. Dans les quartiers, personne n'y participe, à moins qu'un concert d'artistes appréciés et connus localement soit prévu.
} 
d'une tante peu regardante, quand les autres femmes sortent souvent entre célibataires, comme c'était le cas des amies que j'ai accompagnées à plusieurs reprises, parmi lesquelles se trouvait Divina. "Parfois, quand les enfants dorment, je file. Ils ne s'en rendent même pas compte!» commente-t-elle, malicieuse. Elle me dira pendant l'une de nos soirées, alors que nous passons devant le fameux Morro do macaco molhado : "j'y ai été, c'est super pour danser ». Cette confidence est venue rompre le moralisme présent dans les discours généralement employés pour élaborer les images associées à ces lieux.

Sortir danser, c'est prendre le risque de la rencontre avec un homme, car on ne sait pas toujours bien qui l'on va retrouver. Souvent, le contact avec les hommes passe par un frère ou un cousin, qui amènera avec lui des amis. La première partie de la soirée se passe dans un lieu intermédiaire, un bar le plus souvent, comme lors de ma première sortie avec mes amies. En attendant que l'autre groupe arrive, nous dînons et nous commandons des bières. Puis la rencontre a lieu, les hommes arrivent, s'assoient à la table d'à côté, font mine de regarder ailleurs au début, vers l'écran qui diffuse les images du concert d'une Dupla sertaneja, puis entament la discussion en nous offrant à boire. Ils intègrent alors peu à peu notre table, et des couples potentiels se forment. Entre femmes, nous nous levons toutes si l'une d'entre nous manifeste l'envie d'aller aux toilettes et l'accompagnons, pour ne pas rester "seule " avec eux, mais surtout pour échanger nos impressions : " tu as vu comme il est... Et qu'est-ce qu'on fait après ? ». Devant la difficulté de trouver un transport pour aller jusqu'au Sayog, à la sortie de la ville, nous faisons un tour à la fête organisée à l'occasion d'une rencontre de motards dans l'espace construit récemment au bord de la rivière pour les manifestations publiques, la Praça de Eventos, et remontons, toutes les trois avec le frère de l'une d'entre nous, qui fait la cour à Divina depuis le début de la soirée : «je me souviens, quand on était gamins, tu portais un short bien court, je te regardais de haut en bas et pensais, mon Dieu... Un jour elle va voir. (...) On va chez toi !? ». Face à son refus, il réplique, "mais tu es trop compliquée, je me fais toujours refouler avec toi...».

Il arrive aussi qu'on sorte entre femmes pour danser sans avoir rendezvous, comme à l'occasion d'une fête organisée par la faculté de droit. Nous sommes quatre au début de la soirée, et après une heure, alors que l'ambiance tarde à s'installer, Rosimeire décide de rentrer. C'est l'une des premières fois qu'elle sort depuis que son mari l'a quittée et manifestement, sa présence parmi nous n'est pas totalement désirée de sa part, elle s'est plaint pendant tout le parcours nous conduisant jusqu'au lieu de la fête. Une hésitation face au fait de s'exposer publiquement comme femme célibataire quand elle n'assume pas encore ce statut du fait de sa difficulté à accepter la mort de son couple. La soirée commence autour de minuit, quand les étudiants arrivent après leur dernier cours du soir. Avec les deux amies restantes, nous nous rendons sur la piste de danse une fois qu'elle est bien remplie. Il y en a 
toujours une pour porter les sacs à main pendant que les autres dansent. Divina provoque son amie en lui montrant du doigt un homme : " tu veux le prendre celui-là ? ». En attendant des invitations, nous dansons entre nous, à tour de rôle. Les conditions sont alors réunies pour oser séduire, comme on s'y est exercées entre femmes.

Les sorties de ces femmes célibataires, motivées par la possibilité de danser avec les hommes sur les musiques qu'elles écoutent au quotidien, sont des moments qui alimentent leur espoir de faire une rencontre exceptionnelle ou, en fonction des cas, de passer du bon temps. Vaincre la préoccupation du « qu'en dira-t-on », trouver un lieu où aller et des personnes de confiance comme compagnie constituent les prérogatives indispensables pour sortir danser. Il s'agit ensuite de se confronter au regard des hommes, éventuellement de gérer le désir que l'on peut éveiller chez eux et que l'on peut ressentir, tout cela de manière partagée entre femmes, sur le mode de la plaisanterie. Ce moment particulier suscite plusieurs interrogations. Comment s'autorisent-elles à réaliser ce à quoi elles s'étaient préparées en privé, entre femmes ? Quels codes adopter pour danser en public ? Cela exige une adaptation des mouvements du corps, car la manière de danser aux yeux des autres peut constituer un risque de passer pour une femme légère et de mettre ainsi la valeur de sa féminité en jeu. Le fait de vivre ces expériences à plusieurs est aussi une façon de s'assurer de son propre désir d'instaurer ou pas une relation d'intimité avec un homme, plus ou moins durable. Car selon l'histoire de vie de chacune, ces expériences nocturnes peuvent susciter des attentes mais aussi des déceptions chez celles qui n'ont pas abandonné l'idée de construire une relation amoureuse stable, alimentée par la rhétorique que l'on retrouve dans les chansons de musique sertaneja. À travers ces pratiques, les femmes semblent insinuer que si les hommes ne s'intéressent qu'au sexe, alors il est possible de ruser en les attirant par ce biais, mais pas trop près non plus, conscientes du danger que cela peut constituer. S'ils veulent du sérieux, ce sera à leur tour d'en faire la preuve, en matière d'expression des sentiments entre autres. Là aussi, les chansons qu'elles écoutent en quotidien soulignent l'importance de cette dimension. Ces sorties constituent des expériences au cours desquelles elles acceptent de jouer, plus ou moins sérieusement, sur la séduction par la manière dont leurs corps se mettent en mouvement dans ces ambiances musicales.

\section{CONCLUSION}

Le paysage musical du Centre-Ouest brésilien que l'on rencontre dans les quartiers João Francisco et Aeroporto de la ville de Goiás rend possible des lectures de pratiques sociales, de genres musicaux, de sociabilités et de dynamiques du goût musical qui, à l'instar de la région géographique ellemême, ont été peu exploitées par l'anthropologie. 
Ainsi, observer l'univers de celles qui partagent les genres musicaux présentés ici s'est montré révélateur de la resignification d'éléments de la société goiane rurale dans le cadre de la ville, qui vient alimenter une culture urbaine singulière. Les femmes avec lesquelles j'ai cohabité sur le terrain contribuent à valoriser à la fois ces formes musicales comme régime d'expressivité privilégié et les expériences corporelles qui leur sont associées, à tel point que l'esthétique singulière et les particularités sensibles qui s'en dégagent influencent les manières d'habiter ces quartiers.

On remarque qu'à négliger certaines formes musicales brésiliennes dans leurs travaux, les anthropologues peuvent contribuer à maintenir certains préjugés de classe en vigueur dans cette société. Adjectif qualificatif péjoratif désignant de nombreuses formes d'expressivité populaires, mais aussi genre musical à part entière valorisé justement par les classes populaires, le terme brega recouvre au Brésil une ambivalence qui invite le chercheur à se positionner face à la prégnance de certains jugements de valeur, desquels il ne se trouve jamais à l'abri. Ne se limiter qu'à la première acception en ce qui concerne la musique sertaneja constitue un frein à l'appréhension de la spécificité des formes narratives adoptées dans ses chansons. Ces dernières racontent des situations vécues, transmises et partagées de manière particulière dans et sur l'univers féminin, et nous apprennent beaucoup des rapports entre hommes et femmes, mais aussi des rapports entre femmes de Goiás. Ne pas prendre en compte cette spécificité reviendrait surtout à ignorer tout un univers quotidien qui est celui de la plupart des habitants du CentreOuest brésilien.

Comme nous l'avons vu, la musique sertaneja met en rapport de manière singulière différentes réalités sociales, esthétiques et sensibles. Par conséquent, prendre au sérieux les particularités de ces sonorités, les analyser, se révèle une piste féconde pour comprendre l'importance de ce qu'elles peuvent produire en termes de rapports sociaux.

En s'inscrivant dans l'univers social puis en accompagnant l'univers affectif de plusieurs de ces femmes, il est possible de saisir la complexité qui caractérise la place qu'elles réservent à une pratique sociale particulière, l'écoute musicale. En fonction des contextes et des musiques en question, c'est l'aspect romantisé ou sexualisé qui prend le devant. Les dimensions rythmique, mélodique et narrative, suscitent entre autres des réponses érotisées par le corps, comme le rebolado, et la mise en place de véritables chorégraphies de la séduction, mais également une quête de sens au niveau sentimental qui s'inscrit dans le cadre d'histoires de vie singulières. Une expérience qui les amène à participer à des formes de sociabilité féminine de solidarité et de transmission intergénérationnelle sur le mode de la brincadeira, non dépourvues toutefois de tensions, à partir desquelles elles élaborent leurs rapports aux hommes en jouant de leur potentiel de séduction, ceci dans le but de venir combler des absences de leur part. 
Enfin, il apparaît que pour penser ensemble les sonorités, les espaces et les expériences corporelles, il était important d'adopter un paradigme spécifique comme l'a fait entre autres Jean-Paul Thibaud en remettant en cause " l'opposition classique entre sujet sentant et objet senti tant l'un et l'autre ne constituent en fait que les deux faces d'une même médaille» (2010: 208). De fait, j'ai voulu montrer ici que les musiques dont il a été question en tant qu'éléments de l'ambiance sonore des quartiers de Goiás doivent être envisagées comme partie prenante de la construction sensible d'un univers féminin par les femmes qui les habitent.

\section{BIBLIOGRAPHIE}

João Marcos ALEM, Caipira e Country : a Nova Ruralidade Brasileira, Thèse de Doctorat en Sociologie, Universidade de São Paulo, São Paulo, 1996.

Samuel ARAUJO, «Brega: Music and Conflict in Urban Brazil », Latin American Music Review, 9/1, 1988.

Jean-François AUGOYARD, Pas à pas. Essai sur le cheminement quotidien en milieu urbain, Paris, Seuil, 1979.

Jean-François AUGOYARD, «La construction des atmosphères quotidiennes : l'ordinaire de la culture ", Culture et Recherche, n¹14-115, Hiver 2007/2008, pp. 58-60.

Sarah BAKER, "Musique pop et séduction. "It's not about candy. It's about s.e.x." ", Ethnologie française, vol. 40, $\mathrm{n}^{\circ} 1,2010$, pp.31-38.

Jean BAUDRILLARD, De la séduction, Paris, Éditions Galilée, 1979.

Waldenyr CALDAS, Acorde na aurora : música sertaneja e indústria cultural, São Paulo, Ed. Nacional, 1979 ( $2^{\mathrm{a}}$ ed.).

Waldenyr CALDAS, O que é música sertaneja, São Paulo, Brasiliense, 1987.

Antonio CÂNDIDO, Os parceiros do Rio Bonito : estudo sobre o caipira paulista e a transformação dos seus meios de vida, Rio de Janeiro, Ouro sobre Azul, 2010 (1964).

Martha de Ulhôa CARVALHO, « Música Sertaneja em Uberlândia na Década de 1990 (Relato) ", Anais do VIII encontro anual da ANPPOM, Associação Nacional de Pesquisa e Pos-graduação em Música, Articulações entre o discurso musical e o discurso sobre música, Ed. Ilza Nogueira, João Pessoa, 1995 [En ligne]. URL : http://www.anppom.com.br/anais/anaiscongresso_anppom_1995/muscompairel6.htm

Nasr F. CHAUL, Caminhos de Goiás. Da construção da decadência aos limites da modernidade, Goiânia, Editora UCG/Editora UFG, 1997.

Robert DA MATTA, O que faz o brasil, Brasil ?, Rio de Janeiro, Rocco, 1984.

Cécile DAUPHIN et Arlette FARGE, Séduction et sociétés. Approches historiques, Paris, Seuil, 2001.

Tia DE NORA, «Quand la musique de fond entre en action », Terrain, $\mathrm{n}^{\circ} 37,2001$, pp. 75-88.

Françoise DUBOST et Bernadette LIZET, "Pour une ethnologie du paysage ", in Paysage au pluriel. Pour une approche anthropologique des paysages, Paris, Editions de la Maison des Sciences de l'Homme, 1995, pp. 225-240. 
Olivier FERAUD, Voix publiques. Environnements sonores, représentations et usages d'habitation dans un quartier populaire de Naples, Thèse de Doctorat d'Anthropologie Sociale et d'Ethnologie, Paris, EHESS, 2010.

Erving GOFFMAN, Stigmate. Les usages sociaux des handicaps, Paris, Éditions de Minuit, 1975.

François LAPLANTINE, Le social et le sensible. Introduction à une anthropologie modale, Paris, Téraèdre, 2005.

Pierre-Joseph LAURENT, Beautés imaginaires. Anthropologie du corps et de la parenté, Louvain la Neuve, Academia-Bruylant, 2010.

José de Souza MARTINS, «Viola quebrada », Debate \& Crítica, nº 4, 1974, pp. 23-47.

José de Souza MARTINS, «Música sertaneja: a dissimulação na linguagem dos humilhados ", in Capitalismo e tradicionalismo: estudos sobre as contradições da sociedade agrária no Brasil, São Paulo, Pioneira, 1975, pp. 103-161.

Richard PARKER, Corpos, prazeres e paixões : a cultura sexual no Brasil contemporâneo, São Paulo, Best Seller, 1991.

Marina ROUGEON, "-Santé, - Amen! »Récits, chemins et expériences vécues de l'action préventive en santé publique à Goiás, Brésil, Mémoire de Master d'Anthropologie, (sous la direction de M. Thierry Valentin), Université Lumière Lyon 2, 2008.

Marina ROUGEON, «Entrelacer les souvenirs. Mémoires féminines et expériences narratives à Goiás (Brésil) », in François LAPLANTINE (dir.), Mémoires et imaginaires dans les sociétés d'Amérique latine. Harmonie, contrepoints, dissonances, Rennes, Presses Universitaires de Rennes, 2012.

Jorge P. SANTIAGO, «Pratiques musicales urbaines et esthétiques de réélaborations symboliques ", in François LAPLANTINE, Mémoires et imaginaires dans les sociétés d'Amérique latine. Harmonie, contrepoints, dissonances, Rennes, Presses Universitaires de Rennes, 2012.

Jorge P. SANTIAGO, «Paysage musical urbain et perception du sociopolitique au Brésil », Cahiers du Crepal, n 15, 2009, pp. 127-146.

Jorge P. SANTIAGO, «Le paysage urbain de Rio et les terrains du récit. Mémoire vivante des étapes du passé », Second Congrès Bisannuel du GIS - Réseau Amérique Latine, Territoires et Sociétés dans les Amériques, Rennes, 2007.

Jorge P. SANTIAGO, La musique et la ville. Sociabilités et identités urbaines à Campos, Brésil, Paris, L'Harmattan, 1998.

Silvana de SOUZA NASCIMENTO, Faculdades femininas e saberes rurais. Uma etnografia sobre gênero e sociabilidade no interior de Goiás, Thèse de Doctorat en Anthropologie Sociale, Universidade de São Paulo, São Paulo, 2005.

Antônio TEIXEIRA NETO, "Pequena historia da agropecuária goiana », Revista do Instituto Histórico e Geográfico de Goiás, n²0, 2009, pp. 19-58.

Jean-Paul THIBAUD, «La ville à l'épreuve des sens ", in Olivier COUTARD et JeanPierre LEVY (eds.), Écologies urbaines : états des savoirs et perspectives, Paris, Economica Anthropos, 2010, pp. 198-213.

Rachel THOMAS, « La ville charnelle », Cosmopolitiques, n¹5, 2007, pp.111-120.

Rachel THOMAS (dir.), Marcher en ville. Faire corps, prendre corps, donner corps aux ambiances urbaines, Paris, Ed. des Archives Contemporaines, 2010. 
Sidney Valadares PIMENTEL, O chão é o limite - A festa do peão de boiadeiro e a domesticação do sertão, Goiânia, UFG, 1997.

Hermano VIANNA, «Funk et culture populaire carioca », Études historiques, vol.3, n6, 1990, pp. 244-253.

Hermano VIANNA, O mundo funk carioca, Rio de Janeiro, Jorge Zahar, 1988.

Enid YATSUDA, "O caipira e os outros », in Alfredo BOSI (org.), Cultura brasileira, São Paulo, Ática, 1992, pp. 103-113.

RÉSUMÉ : À partir de descriptions issues de mon expérience de terrain, j'aimerais proposer ici une réflexion sur les rapports entre les ambiances sonores qui imprègnent le quotidien de certains quartiers des petites villes du Centre-Ouest brésilien, et les modalités corporelles de déplacement des femmes dans ces espaces. La dimension musicale de ces ambiances, à l'articulation des espaces extérieurs et intérieurs, retiendra particulièrement mon attention ici. En privilégiant des genres musicaux trop peu étudiés par les anthropologues, la musique sertaneja mais aussi la musique brega, le but sera de comprendre le rapport entre leur écoute et les réponses corporelles apportées par les femmes sur le mode de véritables chorégraphies de la séduction. Ce qui me conduira à interroger dans quelle mesure ces expériences sensibles, qui s'inscrivent dans le cadre d'histoires de vie singulières, participent à la construction d'un univers féminin, et ceci en prenant en compte notamment l'importance de différentes formes de sociabilité et de transmission intergénérationnelle. Enfin, plus largement, je chercherai à montrer comment ces expériences contribuent à l'élaboration d'une image modernisée du Brésil rural, m'intéressant par là à la manière dont se construit le rapport entre le rural et l'urbain, le local et le global.

MOTS-CLÉS : Musique sertaneja, ambiance sonore, écoute musicale, expériences et mises en scène corporelles, univers féminin, transmission intergénérationnelle 